\title{
Fibroblast growth factor-1-induced ERK1/2 signaling reciprocally regulates proliferation and smooth muscle cell differentiation of ligament-derived endothelial progenitor cell-like cells
}

\author{
MIKAKO TAKAHASHI $^{1,2}$, NAOTO OKUBO $^{1,3}$, NAOYUKI CHOSA ${ }^{1}$, NORIKO TAKAHASHI ${ }^{1}$, MIHO IBI $^{1,3}$, \\ MASAHARU KAMO $^{1}$, HARUMI MIZUKI ${ }^{2}$, AKIRA ISHISAKI ${ }^{1}$ and SEIKO KYAKUMOTO ${ }^{1}$ \\ ${ }^{1}$ Division of Cellular Biosignal Sciences, Department of Biochemistry; ${ }^{2}$ Division of Oral and Maxillofacial Surgery, \\ Department of Maxillofacial Surgery, School of Dentistry; ${ }^{3}$ Department of Tumor Biology, Institution of \\ Biomedical Science, Iwate Medical University, Nishitokuta, Yahaba-cho, Iwate 028-3694, Japan
}

Received September 15, 2011; Accepted October 25, 2011

DOI: $10.3892 / \mathrm{ijmm} .2011 .847$

\begin{abstract}
The periodontal ligament (PDL) is a fibrous connective tissue located between the tooth root and the alveolar bone. We previously demonstrated that a single cell-derived culture of primarily cultured PDL fibroblasts has the potential to construct an endothelial cell (EC) marker-positive blood vessel-like structure, suggesting that the fibroblastic lineage cells in ligament tissue could act as the endothelial progenitor cells (EPCs), which regenerate to construct a vascular system around the damaged ligament tissue. Moreover, we showed that EPC-like fibroblasts expressed not only EC markers but also smooth muscle cell (SMC) markers. Generally, an interaction between ECs and SMCs regulates blood vessel development and remodeling, and is required for the formation of a mature and functional vascular network. However, the mechanism underlying the SMC differentiation of the ligament-derived EPC-like fibroblasts remains to be clarified. In this study, we showed that suppression of fibroblast growth factor 1 (FGF-1)-induced extracellular signal-regulated kinase $1 / 2$ (ERK1/2) signaling with the MAPK/ERK kinase (MEK) inhibitor U0126 completely abolished the FGF-1-induced proliferation of the ligament-derived EPC-like fibroblasts. In addition, U0126 treatment of FGF-1-stimulated ligamentderived EPC-like fibroblasts significantly induced the SMC differentiation of the cells. Thus, FGF-1-induced ERK1/2 signaling not only promoted the proliferation of the ligamentderived EPC-like fibroblasts, but also suppressed the SMC differentiation of the cells, suggesting that FGF-1 controls the construction of a vascular network around the ligament tissue
\end{abstract}

Correspondence to: Dr Seiko Kyakumoto, Division of Cellular Biosignal Sciences, Department of Biochemistry, Iwate Medical University, 2-1-1 Nishitokuta, Yahaba-cho, Iwate 028-3694, Japan E-mail: kyakumot@iwate-med.ac.jp

Key words: ligament, fibroblast growth factor 1, ERK signaling, proliferation, differentiation, smooth muscle cell by regulating the proliferation and SMC differentiation of the EPC-like cells through ERK-mediated signaling.

\section{Introduction}

Ligament or tendon injuries frequently occur among athletes and workers and are also seen in patients with chronic ligamentosis or tendinosis and ligament or tendon rupture. Ligament and tendon injuries can be significantly disabling, causing lost time from work, physical inactivity, and early retirement from sports or labor. Some ligaments have poor healing ability, which may be caused by the impairment of blood vessels that facilitate the supply and proliferation of mesenchymal progenitor/stem cells to regenerate ligament and tendon fibers to bridge the gap between the damaged ends of ligaments and tendons (1). Thus, angiogenesis can be an essential step in the healing process of ligament and tendon injuries.

The periodontal ligament (PDL) is a fibrous connective tissue that attaches the tooth root to the alveolar bone. The PDL contains a heterogeneous mixture of cell types, including PDL fibroblasts, osteoblasts, vascular endothelial cells (ECs), smooth muscle cells (SMCs), and certain types of nerve cells (2). PDL cells have the capacity to reconstruct the ligament structure in response to oral pathological and physiological environmental alterations such as periodontitis, wounding, and tooth movement due to orthodontic treatment. Tissue reconstruction requires the presence of multipotent progenitor cells or putative stem cells in the PDL. The paravascular zones in the adult PDL contain the progenitors of fibroblasts and mineralized tissueforming cells such as osteoblasts (3). Recently, several studies have indicated that PDL fibroblastic cells share biological characteristics with bone marrow mesenchymal cells, suggesting that the mineralized tissue lineages may have originated from a common progenitor cell (4-7). PDL cells have demonstrated a potential to generate PDL-like tissue in vivo, which suggests that multipotent stem cells are present in the PDL (8).

We previously showed that a swine PDL fibroblast cell line, namely, TesPDL3, could express EC markers and osteoblast markers in addition to PDL markers $(9,10)$. We determined whether PDL fibroblasts could differentiate into putative ECs 
that construct blood vessels with a mature lumen by evaluating their ability to vascularize in a 3-dimensional type I collagen scaffold. We established several single cell-derived cultures (SCDCs) from a primary culture of rat PDL fibroblasts. Intriguingly, each SCDC expressed EC-specific markers, in addition to mesenchymal stem cell- and ligament cell-specific markers. SCDC2 cells, which abundantly expressed the definitive EC marker Tie-2, vigorously constructed a blood vessel structure in a phosphoinositide 3-kinase activation-dependent manner, suggesting that SCDC2 cells must be endothelial progenitor cell (EPC)-like cells (11). Intriguingly, SCDC2 cells expressed not only EC markers but also SMC markers. We also previously demonstrated that human umbilical vein endothelium-derived cells retain the ability to transdifferentiate into SMCs under stimulation by activin $A$ of the transforming growth factor- $\beta$ (TGF- $\beta$ ) superfamily $(12,13)$. However, the molecular mechanisms that control the proliferation and SMC differentiation of ligament-derived EPC-like fibroblasts remain to be elucidated.

Fibroblast growth factors (FGFs) are growth factors involved in the repair and regeneration of tissues (14-16). FGFs were originally identified as a family of proteins that promote fibroblast proliferation, and 22 protein members have been identified. FGFs exert multiple functions by binding to and activating fibroblast growth factor receptors (FGFRs). FGF-induced signaling is mainly mediated via the mitogenactivated protein kinase (MAPK) pathway. Because of their potential biological functions, FGFs have been utilized for the regeneration of damaged tissues, including the skin, blood vessel, muscle, adipose, ligament/tendon, cartilage, bone, tooth, and nerve. MAPKs are protein serine/threonine kinases that transduce extracellular stimulation into a wide range of cellular responses, including cell proliferation, differentiation, and migration (17). c-Jun N-terminal kinase (JNK), extracellular signal-regulated kinase (ERK), and p38MAPK are examples of effector MAPKs. Cell proliferation by FGFs has been reported in many cell types, including ECs, stem cells, and epithelial cells. In a previous report, FGF-2 increased the proliferation of bone marrow-derived mesenchymal stem cells through MAPK/ERK-mediated intracellular signaling (18), which suggests that FGFs might influence the mitosis of mesenchymal progenitor/stem cells through the MAPK/ERK pathway. However, the mechanism by which FGFs-induced MAPK/ERK signaling affects the differentiation of mesenchymal progenitor/stem cells derived from ligament tissues remains to be clarified.

Here, we show how FGF-1-induced ERK activation affects the proliferation and SMC differentiation of the ligament-derived EPC-like SCDC2 cells. Moreover, we simultaneously examined how FGF-1-induced ERK activation affects the EC differentiation of EPC-like cells. This is the first report to show how FGF-1-induced ERK signaling controls not only proliferation but also SMC-differentiation of ligament-derived EPC-like cells.

\section{Materials and methods}

Reagents. Recombinant acidic FGF (FGF-1) was purchased from R\&D Systems, Inc. (Minneapolis, MN, USA). Alamar Blue was obtained from Biosource International (Camarillo, CA USA). Kinase inhibitors, namely, U0126 and
SU5402, were purchased from Calbiochem (Merck KGaA, Darmstadt, Germany).

Cell culture. Isolation of rat PDL fibroblasts and establishment of SCDCs have been previously described (11). Briefly, SCDC2 cells were cultured on type I collagen-coated plastic dishes (Sumilon Celltight Plate, Sumitomo Bakelite Co., Tokyo, Japan) in Ham's F-12 (Sigma Chemicals, St. Louis, MO, USA) supplemented with 2 mM glutamine (x100 solution; Gibco), 20\% fetal bovine serum (FBS; PAA Laboratories, Inc., Ontario, Canada), $10 \mathrm{ng} / \mathrm{ml}$ FGF-1 (R\&D Systems, Inc.), $15 \mu \mathrm{g} / \mathrm{ml}$ heparin (Sigma Chemicals Co., Irvine, UK), $100 \mu \mathrm{g} / \mathrm{ml}$ kanamycin (Meiji Seika Pharma Co., Ltd., Tokyo, Japan), penicillin (Gibco, Carlsbad, CA, USA), and streptomycin (Gibco) in a humidified atmosphere of $95 \%$ air and $5 \% \mathrm{CO}_{2}$ at $37^{\circ} \mathrm{C}$. Heparin was added to achieve optimal FGF-1 activity (19). Subconfluent cells were detached using a trypsin/EDTA solution (0.05\% trypsin, $0.02 \%$ EDTA; Gibco) and subcultured. Cells from passages 13 to 18 were used in the experiments described below. In experiments with chemical inhibitors, each inhibitor was dissolved in dimethylsulfoxide (DMSO) and added to the culture medium, and the same final concentration of DMSO was added to the control culture.

Cell proliferation assay. Evaluation of cell proliferation was carried out using 2 identical methods: measurement of cell number and determination of cell metabolic activity. SCDC2 cells were seeded in standard 12-well plates (Nunc, Roskilde, Denmark) at a density of $2 \times 10^{4}$ cells/well in $2 \mathrm{ml}$ of Ham's F-12 supplemented with $2.5 \%$ FBS and cultured for $24 \mathrm{~h}$. Then, the medium was replaced with media supplemented with various concentrations of FGF-1, and the cells were further cultured for 9-14 days. The medium was changed every 3 days. The cells were detached with trypsin/EDTA solution, and the number of viable cells was determined by the trypan blue (Sigma) exclusion test. The tests were performed in quadruplicates in 3 separate experiments. Cellular metabolic activity was evaluated by the Alamar Blue assay (20). This assay is based on the mitochondrial respiration and utilizes the colorimetric change of the redox indicator Alamar Blue, which is added into the culture medium, from the oxidized form (blue, $\mathrm{A}_{600}$ ) to the reduced form ( $\mathrm{red}, \mathrm{A}_{570}$ ), with mitochondrial reduction potency being proportional to the amount of growing cells. For the Alamar Blue assay, SCDC2 cells were seeded into 96-well plates at a density of $1 \times 10^{3}$ cells/well in $200 \mu 1$ of the medium supplemented with $1 \%$ FBS and cultured. After $24 \mathrm{~h}$, the medium was replaced with media including various concentrations of FGF-1 and further cultured for 9 days, with the medium being changed every 3 days. Various MAPK inhibitors were added $1.5 \mathrm{~h}$ prior to the addition of FGF-1. After 9 days of culture, the medium was replaced with Ham's F-12 containing 10\% Alamar Blue for evaluating the proliferative activity of SCDC2 cells, and the cells were cultured for an additional $4 \mathrm{~h}$. The absorbance of each well was measured using a plate reader (Tosoh Corp., Tokyo, Japan). The data were shown as values of $\mathrm{Abs}_{570}-\mathrm{Abs}_{600}$. Each experiment was repeated 3 times with 6 wells for each point.

Western blotting. The cells were plated onto standard 9-cm plastic dishes at a density of $3 \times 10^{5}$ cells/dish in Ham's F-12 
containing $1 \%$ FBS. After $24 \mathrm{~h}$, FGF-1 was added, and the cells were cultured for the indicated periods. For several experiments, signaling inhibitors were added $2 \mathrm{~h}$ before the addition of FGF-1. Cells were washed twice with PBS, harvested with a cell scraper in $70 \mu 1$ of $6 \mathrm{X}$ SDS sample buffer containing mercaptoethanol and sodium vanadate as a phosphatase inhibitor, and then boiled for $5 \mathrm{~min}$. Equivalent amounts of samples were separated by SDS-PAGE using $12.5 \%$ polyacrylamide gels, and proteins were electrotransferred onto PVDF membranes (Millipore Corp., Bedford, MA, USA). The membranes were blocked with Tris-buffered saline plus $0.1 \%$ Tween-20 (TBS-T) containing 5\% nonfat dry milk or 5\% bovine serum albumin (BSA), as appropriate, for $1 \mathrm{~h}$ at room temperature, and then incubated with primary antibodies overnight at $4^{\circ} \mathrm{C}$. The primary antibodies, i.e., rabbit anti-ERK1/2, rabbit anti-p38MAPK, rabbit anti-JNK, rabbit anti-AKT, rabbit anti-phospho-ERK1/2, rabbit anti-phospho-p38MAPK, rabbit anti-phospho-JNK, mouse anti-phospho-AKT, and rabbit antiglyceraldehyde 3-phosphate dehydrogenase (GAPDH), were obtained from Cell Signaling Technology (Beverly, MA, USA). After being washed with TBS-T, the membranes were incubated with the appropriate horseradish peroxidase-conjugated anti-mouse or anti-rabbit secondary antibody (Cell Signaling). The target proteins were washed with TBS-T and detected by enhanced chemiluminescence with the Amersham ECL ${ }^{\mathrm{TM}}$ Western blotting analysis system (GE Healthcare, Amersham, UK). All other conditions and procedures for each blotting with various antibodies were according to the manufacturer's instructions. The detected blots were photographed using the photo image detection system CL-Cube L (Tohoku Electronic Industrial Co., Ltd.) and densitometrically measured using Image $\mathbf{J}$ (version 1.44). Data were expressed as the ratios of the quantity of the phosphorylated bands to that of the total molecular bands or bands of the reference protein GAPDH.

Quantitative reverse transcriptase-polymerase chain reaction. SCDC2 cells were seeded in $6-\mathrm{cm}$ plastic dishes at a density of $1.3 \times 10^{5}$ cells/dish in $3 \mathrm{ml}$ of Ham's F-12 containing $1 \%$ or $5 \%$ FBS. After $3 \mathrm{~h}$, cells were untreated or treated with various concentrations of U0126, and $2 \mathrm{~h}$ later, stimulated with $16 \mathrm{ng} / \mathrm{ml} \mathrm{FGF-1} \mathrm{and} \mathrm{then} \mathrm{cultured} \mathrm{for} 48 \mathrm{~h}$. Total-RNA was extracted with Isogen reagent (Nippongene, Tokyo, Japan) according to the manufacturer's instructions. First-strand cDNA was synthesized from total-RNA by using the PrimeScript RT Reagent kit (Takara Bio, Inc., Otsu, Japan). Quantitative reverse transcriptase-polymerase chain reaction (qRT-PCR) was performed on a Thermal Cycler Dice Real Time System (Takara) using SYBR ${ }^{\circledR}$ Premix Ex TaqII ${ }^{\text {TM }}$ (Takara) with specific oligonucleotide primers. The mRNA expression levels for SMC and EC markers were normalized to that of the endogenous reference gene GAPDH and were shown as fold-increase or decrease relative to the level of the control sample. The primers used were $\alpha$-SMA, AGCCAGTCGCCATCAGGAAC (sense) and CCGGAGC CATTGTCACACAC (antisense); h1-calponin, ACACTTT AACCGAGGTCCTGCCTA (sense) and CACGCTGGTCG TATTTCTG (antisense); Tie-2, TGCCCAGATATTGGTGT CCTTAAAC (sense) and AGCAGAACAGTCAATTCCTGC GTA (antisense); von Willebrand factor (vWF), CCTACAC TTTGTGGATGTGGAATGAC (sense) and GCGGAAGCCA
TTGGACAGA (antisense); and GAPDH, GGCACAGTCAA GGCTGAGAATC (sense) and ATGGTGGTGAAGACGCC AGTA (antisense).

Immunofluorescence analysis. SCDC2 cells were seeded onto coverslips in 6-cm plastic dishes at a density of $6 \times 10^{3}$ cells $/ \mathrm{cm}^{2}$ $\left(1.3 \times 10^{5}\right.$ cells/dish). After various treatments as described in the preceding section, the cells were cultured. The culture medium was changed every 3 days. Immunocytochemical analysis was performed on day 11. The cells cultured on the coverslips were fixed in 4\% paraformaldehyde for $15 \mathrm{~min}$ and permeabilized with $0.2 \%$ Triton X-100 in PBS for 15 min. The cells were blocked with $3 \%(\mathrm{w} / \mathrm{v})$ BSA in PBS for $1 \mathrm{~h}$, and then incubated with anti- $\alpha$-SMA rabbit polyclonal antiserum (1:200; Abcam Ltd., Cambridge, UK) or anti-calponin rabbit monoclonal antiserum (1:200; Abcam, Ltd.) for $1 \mathrm{~h}$ at room temperature. After being washed with PBS, the cells were incubated with Alexa Fluor 488-conjugated goat anti-rabbit IgG (1:200; Molecular Probes, Leiden, The Netherlands) for $30 \mathrm{~min}$ at room temperature. The cells were washed with PBS and labeled with DAPI (1:1,000; KPL Inc., Gaithersburg, MD, USA) and tetramethylrhodamine B isothiocyanate (TRITC)-conjugated phalloidin (38 $\mu \mathrm{M}$; Sigma-Aldrich, St. Louis, MO, USA) or Alexa Fluor 594 phalloidin (6.6 $\mu \mathrm{M}$; Invitrogen, Paisley, UK). Fluorescent signals were detected and photographed using an Olympus IX71 fluorescence microscope equipped with a DP72 digital camera (Olympus Corp., Tokyo, Japan).

Statistical analysis. Data are presented as means \pm SD. Differences between treatments were evaluated using the Student's t-test for single comparisons. The results shown in all experiments were representative of at least 3 separate experiments. A P value of $<0.05$ was considered statistically significant.

\section{Results}

FGF-1 stimulates cell proliferation of SCDC2 cells through ERK1/2 phosphorylation. SCDC2 cells proliferate vigorously in the presence of FGF-1. Therefore, we decided to investigate the signaling pathway of the growth-stimulating effect of FGF-1 on SCDC2 cells. Cell proliferation was evaluated by the Alarmar Blue assay as described in materials and methods. FGF-1 promoted cell growth in a dose-dependent manner at the concentration range of $2-16 \mathrm{ng} / \mathrm{ml}$ (Fig. 1). Similar results were observed in the determination of the cell number with the trypan blue exclusion test (data not shown). The time course study with $16 \mathrm{ng} / \mathrm{ml} \mathrm{FGF-1} \mathrm{showed} \mathrm{continuous} \mathrm{cell}$ growth until the cells formed a confluent monolayer (data not shown). These results confirmed the growth-stimulatory effect of FGF-1 on SCDC2 cells. Further, we found this stimulatory effect to be completely inhibited by the addition of the FGFR inhibitor SU5402 at $10 \mu \mathrm{M}$ (Fig. 1). These results indicate the relevance of the FGF-1/FGFR complex and its downstream signaling to the growth of SCDC2 cells. Therefore, we examined the level of phosphorylation of the signaling molecules ERK1/2, p38MAPK, and AKT by Western blotting to determine the downstream signaling induced by FGF-1 stimulation. SCDC2 cells were stimulated with $16 \mathrm{ng} / \mathrm{ml} \mathrm{FGF-1,} \mathrm{and}$ samples were obtained at various time points. Stimulation with 


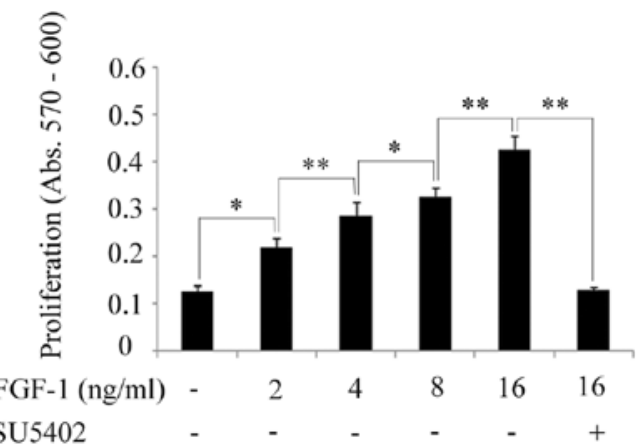

Figure 1. Dose-dependent induction of cell proliferation by FGF-1. SCDC2 cells were plated on 96-well plates ( $1 \times 10^{3}$ cells/well) in $200 \mu 1$ of Ham's F-12 containing $1 \%$ FBS and cultured for $24 \mathrm{~h}$. FGF-1 was added at the indicated final concentrations, and the cells were further cultured; the medium was changed every 3 days. The FGFR inhibitor, SU5402 (10 $\mu \mathrm{M})$, was added in the culture medium $2 \mathrm{~h}$ before FGF-1 stimulation. The Alamar Blue assay was performed on day 9 as described in Materials and methods. The experiment was repeated at least 3 times. Data are represented as mean $\pm \mathrm{SD}$ of 6 wells for each point. ${ }^{*} \mathrm{P}<0.01 ;{ }^{* *} \mathrm{P}<0.05$.

$\mathbf{A}$

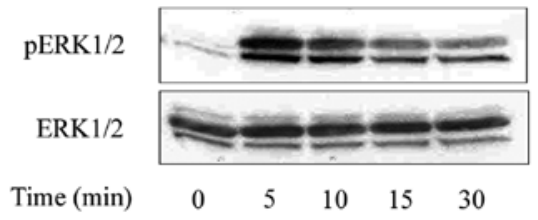

B

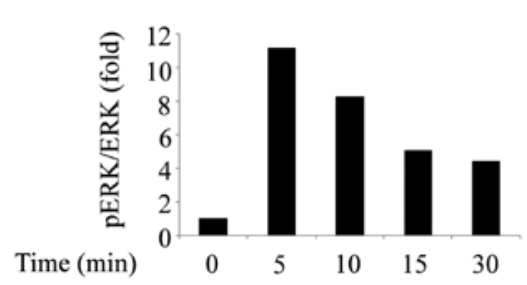

Figure 2. Time course of ERK1/2 phosphorylation. SCDC2 cells were plated and cultured in Ham's F-12 containing 1\% FBS. After $24 \mathrm{~h}$, the cells were stimulated with $16 \mathrm{ng} / \mathrm{ml}$ FGF-1. (A) At various time points after FGF-1 stimulation, cell lysates were recovered and analyzed by Western blotting. (B) The band intensities detected by ECL were densitometrically measured using the Image $\mathbf{J}$ software, and the relative values of pERK/ERK were expressed as compared with the control cells (without FGF-1) at time 0. Experiments were repeated at least 3 times, and similar results were obtained.

FGF-1 upregulated the phosphorylation of ERK1/2, but had no significant effect on total ERK1/2 expression (Fig. 2A). The phospho-ERK1/2 level (pERK/ERK) peaked early (within 5 min) after FGF-1 stimulation, and then gradually decreased (Fig. 2B). The maximum phosphorylation level was more than 10 -fold compared to the unstimulated control level. Moreover, a dose-dependent increase in ERK1/2 phosphorylation was observed with relatively low levels (1-4 ng/ml) of FGF-1 stimulation (data not shown). On the contrary, FGF-1 stimulation only slightly increased ( $<2$-fold) the phosphorylation level of p38MAPK compared to the unstimulated control and did not significantly affect the phosphorylation levels of JNK and
A

a

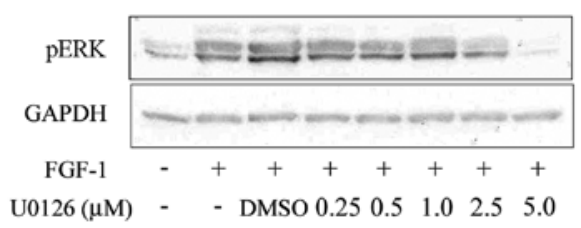

b

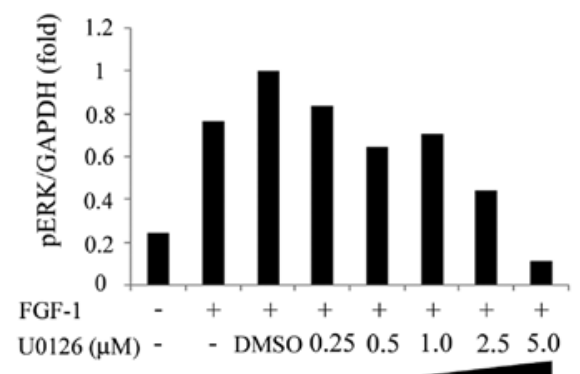

B

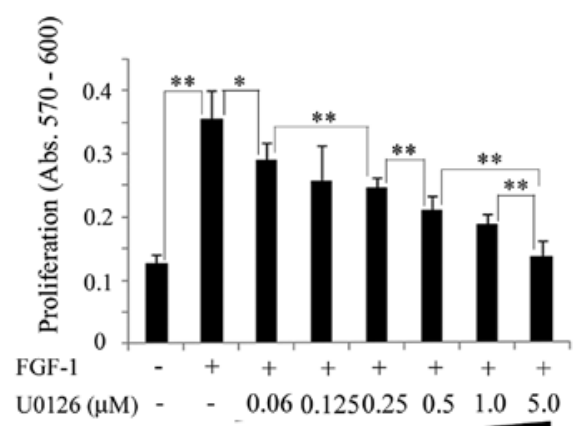

Figure 3. Effects of ERK1/2 signaling inhibition on FGF-1-induced proliferation of SCDC2 cells. (A) SCDC2 cells were plated and cultured in Ham's F-12 containing $1 \%$ FBS. After $24 \mathrm{~h}$, the cells were untreated or treated with various concentrations of U0126, and $2 \mathrm{~h}$ later, stimulated with $16 \mathrm{ng} / \mathrm{ml} \mathrm{FGF-1.}$ After 5 min of FGF-1 stimulation, cell lysates were recovered and ERK1/2 phosphorylation was examined by Western blotting. (B) The cells were plated on 96-well plates and cultured in the presence of $1 \%$ FBS. After $24 \mathrm{~h}$, various concentrations of U0126 were added, and $2 \mathrm{~h}$ later, cells were treated with $16 \mathrm{ng} / \mathrm{ml}$ FGF-1 and further cultured. The medium in all wells was changed every 3 days with fresh medium containing U0126 and FGF-1. The Alamar Blue cell proliferation assay was performed on day 9 as described in Materials and methods. Experiments were repeated at least 3 times. In (B), data are presented as means \pm SD of 6 -wells for each point. ${ }^{*} \mathrm{P}<0.01 ;{ }^{* *} \mathrm{P}<0.05$.

AKT (data not shown). These results suggest that the ERK1/2 signaling pathway is the main mediator of the proliferative effects of FGF-1 on SCDC2 cells.

To confirm this finding, we investigated the effect of MEK-ERK signaling inhibition by adding the MEK inhibitor U0126 to the culture medium. We first examined the ERK1/2 phosphorylation in the presence of various concentrations $(0.25-5 \mu \mathrm{M})$ of U0126. Fig. 3A shows that pretreatment of SCDC2 cells with U0126 in a dose-dependent manner inhibited the phosphorylation of ERK1/2 induced by FGF-1. The phosphorylation level of ERK1/2 at $5 \mu \mathrm{M}$ U0126 was similar to or rather lower than that of the unstimulated cells (Fig. 3A-b). The solvent for U0126, DMSO, which was used as a control, had almost no significant effect on the level of phosphorylation 
$\mathbf{A}$

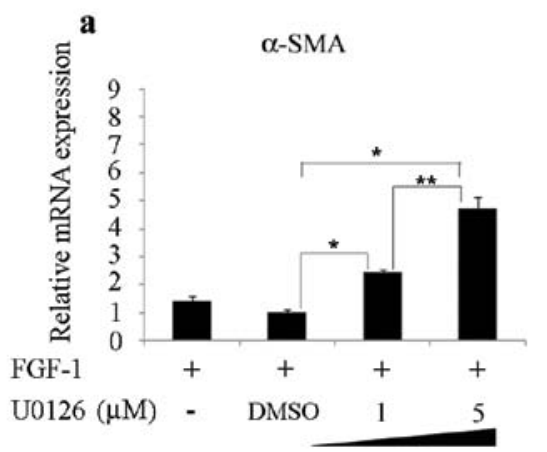

B

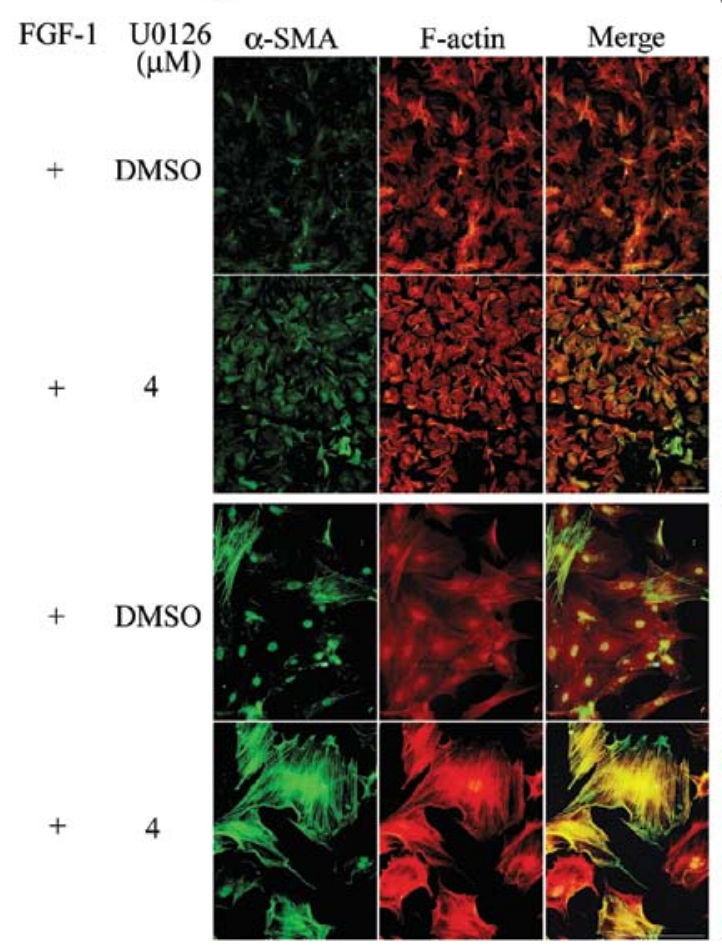

b

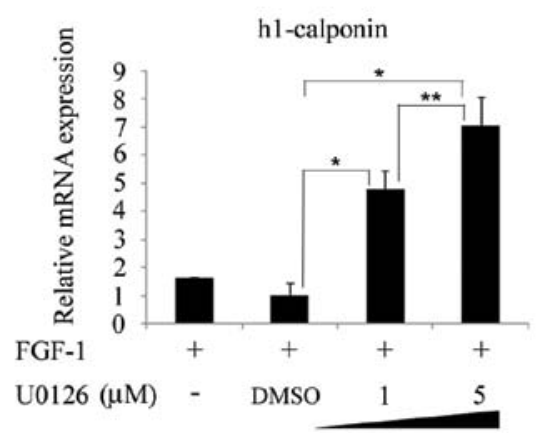

b
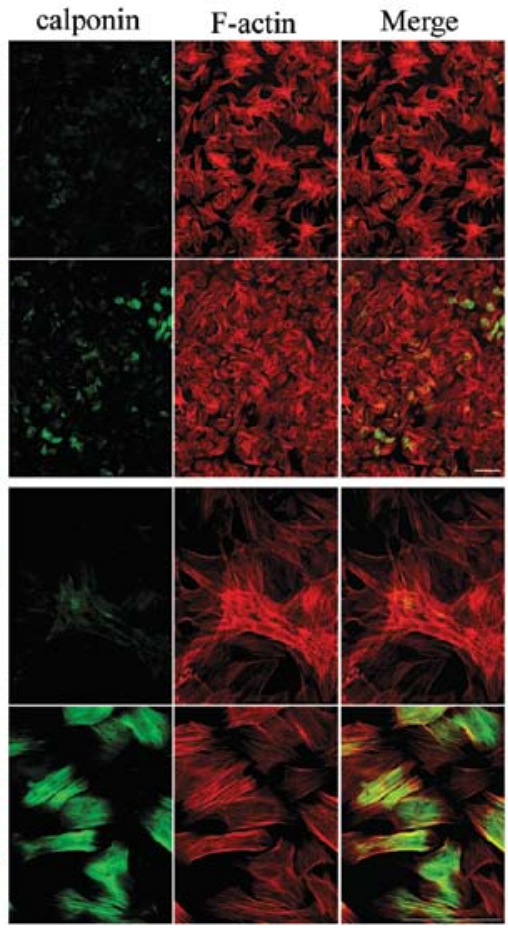

Figure 4. Effects of ERK1/2 signaling inhibition under FGF-1 stimulation on the expression of SMC markers in SCDC2 cells. (A) SCDC2 cells were plated onto 6-cm dishes in Ham's F-12 containing 5\% FBS. After $3 \mathrm{~h}$, cells were untreated or treated with various concentrations of U0126 and, $2 \mathrm{~h}$ later, stimulated with $16 \mathrm{ng} / \mathrm{ml}$ FGF-1. After further cultivation for $48 \mathrm{~h}$, total-RNA was isolated, and qRT-PCR of SMC marker mRNAs (a, $\alpha$-SMA; b, h1-calponin) was performed on a Thermal Cycler Dice Real Time System as described in Materials and methods. GAPDH was analyzed as an endogenous reference mRNA. Data are represented as the means \pm SD of 4 dishes for each point. ${ }^{*} \mathrm{P}<0.01 ;{ }^{* * *} \mathrm{P}<0.05$. (B) SCDC2 cells were seeded onto coverslips in 6 -cm plastic dishes in $1 \%$ FBS-containing Ham's F-12. After various treatments as described in (A), immunocytochemical analysis was performed on day 11. The cells on the coverslips were fixed in $4 \%$ paraformaldehyde, permeabilized with $0.2 \%$ Triton X-100, and blocked with $1 \%$ BSA in PBS. Cells were then incubated with anti- $\alpha$-SMA rabbit polyclonal antiserum (a; 1:200) and anti-calponin rabbit monoclonal antibody (b; 1:200). After being incubated with Alexa Fluor 488-conjugated goat anti-rabbit IgG (1:200), the cells were stained with TRITC-labeled phalloidin, and fluorescent signals were detected and photographed. The details are described in Materials and methods. DMSO was used as solvent for U0126. Original magnification: upper 2 rows, $\mathrm{x} 40$; lower 2 rows, $\mathrm{x} 200$. Scale bar, $50 \mu \mathrm{m}$.

of ERK1/2 in both unstimulated (data not shown) and FGF-1stimulated SCDC2 cells (Fig. 3A). Next, we examined the effect of U0126 on FGF-1-induced cell proliferation. U1026 inhibited the FGF-1-induced cell proliferation of SCDC2 cells (Fig. 3B) in a dose-dependent manner at the concentration range of 0.06-5 $\mu \mathrm{M}$, with $5 \mu \mathrm{M}$ U0126 almost completely inhibiting the cell proliferation induced by $16 \mathrm{ng} / \mathrm{ml}$ FGF-1. These results indicate that FGF-1 promotes SCDC 2 cell proliferation mediated by FGFR and the downstream MEK-ERK1/2 signaling pathway.

Inhibition of FGF-1-induced MEK-ERK1/2 signaling induces the differentiation of SCDC2 cells into SMC-like cells. To investigate the effects of FGF-1-induced MEK-ERK1/2 signaling on the expression status of EC- and SMC-specific markers in FGF-1-stimulated SCDC2 cells, the cells were pretreated with various concentrations $(0.06-5 \mu \mathrm{M})$ of U0126, and then stimulated with FGF-1. The mRNA and protein expression levels of the SMC-specific markers $\alpha$-SMA and h1-calponin in the U0126-treated SCDC2 cells under FGF-1 stimulation were evaluated by qRT-PCR and immunocytochemical analyses, respectively. U0126 treatment increased the mRNA expression levels of both $\alpha$-SMA (Fig. 4A-a) and h1-calponin (Fig. 4A-b) in a dose-dependent manner at the concentration range of $1-5 \mu \mathrm{M}$ compared to the untreated or DMSO-treated control cells. Immunocytochemical analysis (Fig. 4B) revealed that the presence of $4 \mu \mathrm{M}$ U0126 in SCDC2 cell culture under $10 \mathrm{ng} / \mathrm{ml}$ FGF-1 stimulation enhanced $\alpha$-SMA staining compared to the DMSO-treated control cells 
A

a

B
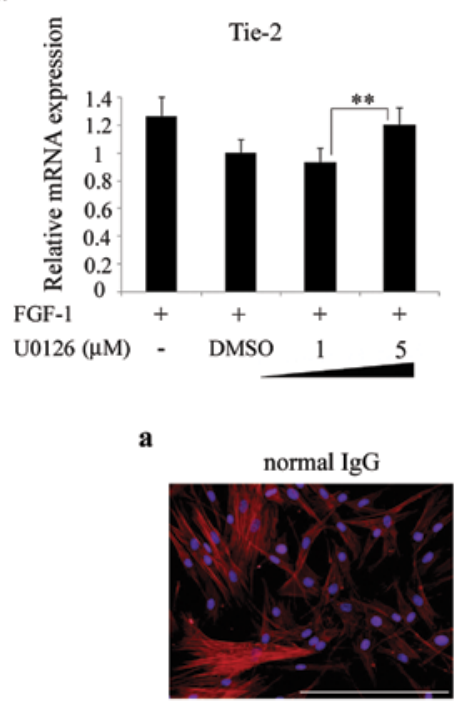

b

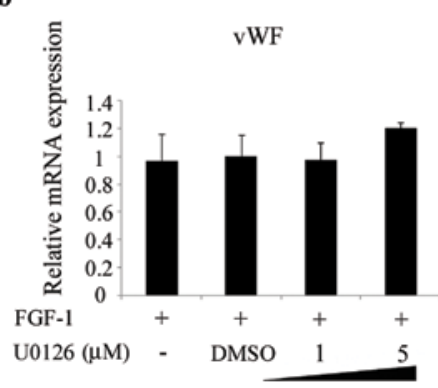

b

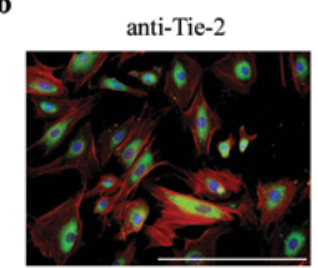

Figure 5. Effects of ERK1/2 signaling inhibition on the expression of EC markers in SCDC2 cells under FGF-1 stimulation. (A) SCDC2 cells were plated onto 6-cm culture dishes in Ham's F-12 containing 5\% FBS. After $3 \mathrm{~h}$, cells were untreated or treated with various concentrations of U0126, and $2 \mathrm{~h}$ later, stimulated with $16 \mathrm{ng} / \mathrm{ml}$ FGF. After $48 \mathrm{~h}$, total-RNA was isolated and qRT-PCR of EC marker mRNAs (a) Tie-2; (b) vWF was performed as described in the legend to Fig. 4. Data are represented as mean \pm SD of 4 dishes for each point. ${ }^{*} \mathrm{P}<0.01{ }^{*}{ }^{* *} \mathrm{P}<0.05$. (B) SCDC2 cells were plated onto individual wells of type I collagen-coated 8-chamber slides in 5\% FBS-containing Ham's F-12 at a density of $1 \times 10^{4}$ cells/well. After $48 \mathrm{~h}$ of cultivation, the cells were fixed in $4 \%$ paraformaldehyde, permeabilized with $0.2 \%$ Triton X-100, and blocked with $1 \%$ BSA in PBS. Cells were then incubated with anti-Tie-2 rabbit polyclonal antiserum $(1: 50 ; \mathrm{b}$ ) or the same protein amount of normal rabbit IgG as a control (a) for $1 \mathrm{~h}$. After being washed with $0.2 \%$ Triton X-100 in PBS, the cells were incubated with Alexa Fluor 488-conjugated goat anti-rabbit $\operatorname{IgG}(1: 1,000)$. The cells were then washed and stained with DAPI and Alexa Fluor 594 phalloidin $(6.6 \mu \mathrm{M}$; Invitrogen, Paisley, UK). Fluorescent signals were detected and photographed as described in Materials and methods. Scale bar, $50 \mu \mathrm{m}$.

(Fig. 4B-a). A similar result was observed in the staining of calponin (Fig. 4B-b). Both $\alpha$-SMA and calponin showed stronger staining with increasing concentrations of U0126 (data not shown). On the other hand, U0126 treatment did not significantly affect the mRNA expression of the EC-specific markers Tie-2 and vWF in FGF-1-stimulated SCDC2 cells (Fig. 5A). In addition, the results of immunocytochemistry studies revealed that treatment with up to $5 \mu \mathrm{M}$ U0126 under FGF-1 stimulation did not significantly affect the expression levels of these proteins (data not shown), although SCDC2 actually expresses Tie-2 protein (green in Fig. 5B-b) and vWF (data not shown). These results suggest that the large population of SCDC2 cells maintained an EC-like characteristic, even if FGF-induced ERK1/2 signaling was inhibited by U0126, and that FGF-1-induced ERK1/2 signaling suppresses the SMC-like differentiation of SCDC2 cells.

Inhibition of FGF-1-induced MEK-ERK1/2 signaling induces actin polymerization in SCDC 2 cells. Immunocytochemical analysis of $\alpha$-SMA (Fig. 4B-a) further revealed that U0126 treatment under FGF-1 stimulation induced the polymerization of $\alpha$-SMA. Images captured at higher magnification (lower 2 rows) show the clearly stained polymerized $\alpha$-SMA fibers (green, bottom left) in the U0126-treated SCDC2 cells. The U0126-induced actin polymerization suggests that the altered cells harbor SMC-like characteristics.

\section{Discussion}

FGF intracellular signaling is mainly mediated by the MAPK pathway. JNK, ERK, and p38MAPK are examples of effector MAPKs. Among them, FGF-2-stimulated ERK1/2 induces the proliferation of ECs and the differentiation of progenitor stem cells into ECs, SMCs, and neural cells (21-23). As shown in Figs. 1-3, FGF-1 positively upregulated the proliferation of ligament-derived EPC-like SCDC2 cells mainly through the ERK1/2-mediated pathway, which is in agreement with the findings of previous reports. Analysis of the activation of other MAPKs, i.e., p38 MAPK and JNK, showed that FGF-1 induced low level (<2-fold) p38 MAPK phosphorylation, whereas no significant activation of JNK was observed (data not shown).

FGF/ERK signaling controls the pluripotency and lineage specification of different states of stem cells, including separation of the pluripotent epiblast and primitive endoderm in the blastocyst during early embryonic developmental stages (24). In addition, autocrine FGF-induced ERK1/2 signaling occurs in the embryonic stem (ES) cells and is required to initiate the differentiation of the cells into neural lineage $(25,26)$. FGF-4/ ERK signaling plays important roles in the ES cell commitment into the endoderm or germ lineage (27). Intriguingly, Ying et al found that addition of the FGFR inhibitor SU5402 or the ERK (MEK) inhibitor PD184352 could maintain mouse ES cells in an undifferentiated state in the presence of leukemia inhibitory factor (28). On the other hand, FGF may indirectly maintain human ES cells in an undifferentiated state by inducing TGF- $\beta 1$ and activin A secretion in their feeder cells (29). Thus, FGF-stimulated ERK intracellular signaling plays various and, sometimes, contrasting roles in early ES cells. FGF/ERK maintains pluripotency and drives lineage differentiation. In contrast, in adult stem cells such as bone marrow-derived mesenchymal stem cells, FGF-2 induces self-renewal of ES cells and is involved in the maintenance of their multi-lineage differentiation potential (30) through ERK-mediated cellular signaling (31). 
The important point to note is how cells coordinate proliferation and differentiation during early development and tissue regeneration. In general, growth and proliferation are poorly compatible with differentiation, and proliferation/differentiation switches have been demonstrated in different cell types (32-34). Upon differentiation of the progenitor/stem cells during regeneration or reconstruction of damaged tissues, the expression of the proliferation module such as transcription factors and intracellular signals for cell growth is uniformly suppressed, suggesting that proliferation and differentiation modules may correspond to 2 alternative states of the molecular network (35). The molecular mechanisms that control the proliferation/differentiation switch in progenitor/stem cells, which would be an invaluable information for establishing novel progenitor/stem cell therapies for regenerative medicine remain to be clarified. Self-renewal and differentiation of vascular progenitor cells is necessary especially for the regeneration of damaged ligament tissues; however, the intracellular signals involved in the control of these events are still unknown.

In the present study, inhibition of FGF-induced phosphorylation of ERK1/2 by U0126 treatment suppressed the proliferation of SCDC2 cells (Fig. 3) and induced their differentiation into SMCs (Fig. 4). As shown in Fig. 3A, U0126 inhibited FGF-1induced ERK1/2 phosphorylation in a dose-dependent manner. Intriguingly, the status of ERK1/2 phosphorylation in the SCDC2 cells was parallel to that of the proliferative activity of the cells. On the other hand, the status of ERK $1 / 2$ phosphorylation was inversely correlated with the expression status of SMC markers in the cells. These results suggest that FGF-induced ERK1/2 signaling reciprocally regulates the proliferation and SMC differentiation of ligament-derived EPC-like cells, thus implicating ERK1/2 as a key molecule in the regulation of the proliferation/SMC-differentiation switch in the cells. Intriguingly, as shown in Fig. 4B-a, U0126 treatment induced $\alpha$-SMA polymerization in SCDC2 cells under FGF-1 stimulation, suggesting that FGF-1-induced ERK1/2 signaling controls the dynamics of the cytoskeletal network. Actin polymerization is necessary for contraction and tension development in SMCs (36). Moreover, the upregulation of h1-calponin (Figs. 4A-b and B-b), which acts as an actin-myosin II-regulating protein, strongly suggested that the induced SMC might act as a functional cell. Therefore, it is plausible that the inhibition of ERK1/2-mediated signaling in SCDC2 cells under FGF-1 stimulation might promote not only the expression of SMC-marker genes but also the emergence of SMC-like contractile characteristics in the cells.

On the other hand, as shown in Fig. 5, the expression levels of EC markers were almost constant, whereas those of SMC markers were significantly upregulated in the U0126-treated SCDC2 cells. This may indicate that a large population of SCDC2 cells maintained an EC-like characteristic, even if FGF-1-induced ERK1/2 signaling was inhibited by U0126. Thus, ERK1/2 signal disruption might not sufficiently induce the terminal SMC differentiation of EPC-like SCDC2 cells.

Previous reports demonstrated that TGF- $\beta$ upregulated the expression of SMC markers in vascular SMCs through activation of the transcription factor Krüppel-like factor 4 (KLF4) (37). In addition, the TGF- $\beta$-induced transcription factor myocardin transactivated multiple SMC-specific transcrip- tional regulatory elements in undifferentiated mesenchymal cells (38). The downregulation of ERK1/2 activity in FGF-1stimulated SCDC 2 cells may cause TGF- $\beta$ secretion, and the secreted TGF- $\beta$, in turn, may induce the SMC differentiation of SCDC2 cells in an autocrine fashion. An investigation is under way in our laboratory on whether TGF- $\beta$ signaling plays a crucial role in the terminal SMC differentiation of ligamentderived EPC-like cells.

Thus, FGF-1-induced ERK1/2 signaling not only promotes the proliferation of ligament-derived EPC-like fibroblasts, but also suppresses the SMC differentiation of EPC-like cells. The FGF-1-induced ERK1/2 activity gradient controls the proliferation/SMC-differentiation switch of the cells. These results suggest that FGF-1 controls the vascular network construction around the ligament tissue by regulating the proliferation and SMC differentiation of EPC-like cells through ERK-mediated signaling. Our findings provide new insights toward the establishment of new cell therapeutic methods for ligament regeneration by constructing a vascular network composed of ECs and SMCs for nutrient delivery to the damaged ligament tissue with the ligament-derived EPC-like fibroblasts.

\section{Acknowledgements}

This study was supported in part by Grants-in-Aid for Scientific Research (no. 22791935 to N.O., no. 22791936 to N.T., no. 23592896 to A.I., no. 22592076 to M.K., no. 19791370 to N.C.) from the Ministry of Education, Culture, Sports, Science, and Technology of Japan; the Open Research and High-Tech Research Project from the Ministry of Education, Culture, Sports, Science, and Technology of Japan; a Grant-in-Aid for Strategic Medical Science Research Center from the Ministry of Education, Culture, Sports, Science, and Technology of Japan, 2010-2014; and grants from the Keiryokai Research Foundation (no. 100 to N.C., 2008; no. 103 to A.I., 2009).

\section{References}

1. Ju YJ, Tohyama H, Kondo E, Yoshikawa T, Muneta T, Shinomiya K and Yasuda K: Effects of local administration of vascular endothelial growth factor on properties of the in situ frozen-thawed anterior cruciate ligament in rabbits. Am J Sports Med 34: 84-91, 2006.

2. Hou LT and Yaeger JA: Cloning and characterization of human gingival and periodontal ligament fibroblasts. J Periodontol 64: 1209-1218, 1993.

3. McCulloch CA: Progenitor cell populations in the periodontal ligament of mice. Anat Rec 211: 258-262, 1985.

4. Pitaru S, Pritzki A, Bar-Kana I, Grosskopf A, Savion N and Narayanan AS: Bone morphogenetic protein 2 induces the expression of cementum attachment protein in human periodontal ligament clones. Connect Tissue Res 43: 257-264, 2002.

5. Nakamura T, Yamamoto M, Tamura M and Izumi Y: Effects of growth/differentiation factor-5 on human periodontal ligament cells. J Periodontal Res 38: 597-605, 2003.

6. Trubiani O, Isgro A, Zini N, Antonucci I, Aiuti F, Di Primio R, Nanci A, Caputi S and Paganelli R: Functional interleukin-7/ interleukin-7Ralpha, and SDF-1alpha/CXCR4 are expressed by human periodontal ligament derived mesenchymal stem cells. J Cell Physiol 214: 706-713, 2008.

7. Shi S, Bartold PM, Miura M, Seo BM, Robey PG and Gronthos S: The efficacy of mesenchymal stem cells to regenerate and repair dental structures. Orthod Craniofac Res 8: 191-199, 2005.

8. Seo BM, Miura M, Gronthos S, Bartold PM, Batouli S, Brahim J, Young M, Robey PG, Wang CY and Shi S: Investigation of multipotent postnatal stem cells from human periodontal ligament. Lancet 364: 149-155, 2004. 
9. Ibi M, Ishisaki A, Yamamoto M, Wada S, Kozakai T, Nakashima A, Iida J, Takao S, Izumi Y, Yokoyama A and Tamura M: Establishment of cell lines that exhibit pluripotency from miniature swine periodontal ligaments. Arch Oral Biol 52: 1002-1008, 2007.

10. Shirai K, Ishisaki A, Kaku T, Tamura M and Furuichi Y: Multipotency of clonal cells derived from swine periodontal ligament and differential regulation by fibroblast growth factor and bone morphogenetic protein. J Periodontal Res 44: 788-795, 2009.

11. Okubo N, Ishisaki A, Iizuka T, Tamura M and Kitagawa $\mathrm{Y}$ : Vascular cell-like potential of undifferentiated ligament fibroblasts to construct vascular cell-specific marker-positive blood vessel structures in a PI3K-activation-dependent manner. J Vasc Res 47: 369-383, 2010

12. Ishisaki A, Hayashi H, Li AJ and Imamura T: Human umbilical vein endothelium-derived cells retain potential to differentiate into smooth muscle-like cells. J Biol Chem 278: 1303-1309, 2003.

13. Ishisaki A, Tsunobuchi H, Nakajima K and Imamura T: Possible involvement of protein kinase $\mathrm{C}$ activation in differentiation of human umbilical vein endothelium-derived cell into smooth muscle-like cell. Biol Cell 96: 499-508, 2004.

14. Yun YR, Won JE, Jeon E, Lee S, Kang W, Jo H, Jang JH, Shin US and Kim HW: Fibroblast growth factors: biology, function, and application for tissue regeneration. J Tissue Eng 2010: 218142, 2010.

15. Zakrzewska M, Marcinkowska E and Wiedlocha A: FGF-1: from biology through engineering to potential medical applications. Crit Rev Clin Lab Sci 45: 91-135, 2008.

16. Robinson ML: An essential role for FGF receptor signaling in lens development. Semin Cell Dev Biol 17: 726-740, 2006.

17. Cargnello M and Roux PP: Activation and function of the MAPKs and their substrates, the MAPK-activated protein kinases Microbiol Mol Biol Rev 75: 50-83, 2011.

18. Bocelli-Tyndall C, Zajac P, Di Maggio N, Trella E, Benvenuto F, Iezzi G, Scherberich A, Barbero A, Schaeren S, Pistoia V, Spagnoli G, Vukcevic M, Martin I and Tyndall A: Fibroblast growth factor 2 and platelet-derived growth factor, but not platelet lysate, induce proliferation-dependent, functional class II major histocompatibility complex antigen in human mesenchymal stem cells. Arthritis Rheum 62: 3815-3825, 2010.

19. Harmer NJ: Insights into the role of heparan sulfate in fibroblast growth factor signaling. Biochem Soc Trans 34: 442-445, 2006.

20. Fields RD and Lancaster MV: Dual attribute continuous monitoring of cell proliferation/cytotoxicity. Am Biotechnol Lab 11: 48-50, 1993.

21. Lee JG and Kay EP: PI3-kinase/Rac1 and ERK1/2 regulate FGF-2mediated cell proliferation through phosphorylation of p27 at Ser10 by KIS and at Thr187 by Cdc25A/Cdk2. Invest Ophthalmol Vis Sci 52: 417-426, 2011

22. Hutson MR, Zeng XL, Kim AJ, Antoon E, Harward S and Kirby ML: Arterial pole progenitors interpret opposing FGF/ BMP signals to proliferate or differentiate. Development 137 : 3001-3011, 2010

23. Sato T, Shimazaki T, Naka H, Fukami S, Satoh Y, Okano H, Lax I Schlessinger J and Gotoh N: FRS2a regulates Erk levels to control a self-renewal target Hes1 and proliferation of FGF-responsive neural stem/progenitor cells. Stem Cells 28: 1661-1673, 2010.
24. Lanner F and Rossant J: The role of FGF/Erk signaling in pluripotent cells. Development 137: 3351-3360, 2010.

25. Kunath T, Saba-El-Leil MK, Almousailleakh M, Wray J, Meloche S and Smith A: FGF stimulation of the Erk1/2 signaling cascade triggers transition of pluripotent embryonic stem cells from self-renewal to lineage commitment. Development 134 : 2895-2902, 2007

26. Stavridis MP, Lunn JS, Collins BJ and Storey KG: A discrete period of FGF-induced Erk1/2 signalling is required for vertebrate neural specification. Development 134: 2889-2894, 2007.

27. Lanner F, Lee KL, Sohl M, Holmborn K, Yang H, Wilbertz J, Poellinger L, Rossant J and Farnebo F: Heparan sulfation dependent fibroblast growth factor signaling maintains embryonic stem cells primed for differentiation in a heterogeneous state. Stem Cells 28: 191-200, 2010.

28. Ying QL, Wray J, Nichols J, Batlle-Morera L, Doble B, Woodgett J, Cohen P and Smith A: The ground state of embryonic stem cell self-renewal. Nature 453: 519-523, 2008.

29. Greber B, Lehrach H and Adjaye J: Fibroblast growth factor 2 modulates transforming growth factor beta signaling in mouse embryonic fibroblasts and human ESCs (hESCs) to support hESC self-renewal. Stem Cells 25: 455-464, 2007.

30. Tsutsumi S, Shimazu A, Miyazaki K, Pan H, Koike C, Yoshida E, Takagishi K and Kato Y: Retention of multilineage differentiation potential of mesenchymal cells during proliferation in response to FGF. Biochem Biophys Res Commun 288: 413-419, 2001.

31. Choi SC, Kim SJ, Choi JH, Park CY, Shim WJ and Lim DS: Fibroblast growth factor-2 and -4 promote the proliferation of bone marrow mesenchymal stem cells by the activation of the PI3K-Akt and ERK1/2 signaling pathways. Stem Cells Dev 17: 725-736, 2008

32. Chen JF, Mandel EM, Thomson JM, Wu Q, Callis TE, Hammond SM, Conlon FL and Wang DZ: The role of microRNA-1 and microRNA-133 in skeletal muscle proliferation and differentiation. Nat Genet 38: 228-233, 2006.

33. Conti L, Sipione S, Magrassi L, Bonfanti L, Rigamonti D, Pettirossi V, Peschanski M, Haddad B, Pelicci P, Milanesi G, Pelicci $G$ and Cattaneo E: Shc signaling in differentiating neural progenitor cells. Nat Neurosci 4: 579-586, 2001.

34. Dugan LL, Kim JS, Zhang Y, Bart RD, Sun Y, Holtzman DM and Gutman DH: Differential effects of cAMP in neurons and astrocytes. Role of B-raf. J Biol Chem 274: 25842-25848, 1999.

35. Xia K, Xue H, Dong D, Zhu S, Wang J, Zhang Q, Hou L, Chen H, Tao R, Huang Z, Fu Z, Chen YG and Han JD: Identification of the proliferation/differentiation switch in the cellular network of multicellular organisms. PLoS Comput Biol 2: 1483-1497, 2006.

36. Gunst SJ and Zhang W: Actin cytoskeletal dynamics in smooth muscle: a new paradigm for the regulation of smooth muscle contraction. Am J Physiol Cell Physiol 295: C576-C587, 2008.

37. King KE, Iyemere VP, Weissberg PL and Shanahan CM: Krüppellike factor 4 (KLF4/GKLF) is a target of bone morphogenetic proteins and transforming growth factor 1 in the regulation of vascular smooth muscle cell phenotype. J Biol Chem 278: 11661-11669, 2003

38. Xie WB, Li Z, Miano JM, Long X and Chen SY: Smad3-mediated myocardin silencing: mechanism governing the initiation of smooth muscle differentiation. J Biol Chem 286: 15050-15057, 2011. 